\title{
Sox2 is not required for melanomagenesis, melanoma growth and melanoma metastasis in vivo
}

Article in Oncogene · April 2017

DOI: $10.1038 /$ onc. 2017.53

CITATIONS

0

14 authors, including:

\section{Valeriana Cesarini}

Ospedale Pediatrico Bambino Gesù

10 PUBLICATIONS 69 CITATIONS

SEE PROFILE

Emmanuele A. Jannini

University of Rome Tor Vergata

301 PUBLICATIONS 7,444 CITATIONS

SEE PROFILE
READS

20

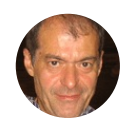

Pellegrino Rossi

University of Rome Tor Vergata

90 PUBLICATIONS $\quad 4,077$ CITATIONS

SEE PROFILE

\section{Susanna Dolci}

University of Rome Tor Vergata

94 PUBLICATIONS 3,980 CITATIONS

SEE PROFILE

Some of the authors of this publication are also working on these related projects:

Project Neuro-oncology and cancer stem cell-like in glioblastoma View project 


\title{
SHORT COMMUNICATION
}

\section{Sox 2 is not required for melanomagenesis, melanoma growth and melanoma metastasis in vivo}

\author{
V Cesarini ${ }^{1,7}$, E Guida ${ }^{1,7}, \mathrm{~F}$ Todaro ${ }^{1}$, S Di Agostino ${ }^{2}$, V Tassinari ${ }^{1}$, S Nicolis ${ }^{3}$, R Favaro ${ }^{3}$, S Caporali $^{4}$, PM Lacal ${ }^{4}$, E Botti $^{5}$, A Costanzo $^{6}$, \\ P Rossi ${ }^{1}$, EA Jannini ${ }^{5}$ and $S$ Dolci ${ }^{1}$
}

\begin{abstract}
Melanoma is a dangerous form of skin cancer derived from the malignant transformation of melanocytes. The transcription factor SOX2 is not expressed in melanocytes, however, it has been shown to be differentially expressed between benign nevi and malignant melanomas and to be essential for melanoma stem cell maintenance and expansion in vitro and in xenograft models. By using a mouse model in which BRaf ${ }^{\mathrm{V} 600 \mathrm{E}}$ mutation cooperates with Pten loss to induce the development of metastatic melanoma, we investigated if Sox2 is required during the process of melanomagenesis, melanoma growth and metastasis and in the acquisition of resistance to BRAF inhibitors (BRAFi) treatments. We found that deletion of Sox2 specifically in Pten null and BRafV600E-expressing melanocytes did not prevent tumor formation and did not modify the temporal kinetics of melanoma occurrence compared to Sox 2 wt mice. In addition, tumor growth was similar between Sox 2 wt and Sox2 deleted (del) melanomas. By querying publicly available databases, we did not find statistically significant differences in SOX2 expression levels between benign nevi and melanomas, and analysis on two melanoma patient cohorts confirmed that Sox2 levels did not significantly change between primary and metastatic melanomas. Melanoma cell lines derived from both Sox 2 genotypes showed a similar sensitivity to vemurafenib treatment and the same ability to develop vemurafenib resistance in long-term cultures. Development of vemurafenib resistance was not dependent on SOX2 expression also in human melanoma cell lines in vitro. Our findings exclude an oncogenic function for Sox2 during melanoma development and do not support a role for this transcription factor in the acquisition of resistance to BRAFi treatments.
\end{abstract}

Oncogene advance online publication, 3 April 2017; doi:10.1038/onc.2017.53

\section{INTRODUCTION}

Sox2 belongs to the SoxB1 subgroup of Sox transcription factors and consists of a single exon gene mapping on mouse chromosome 3 and encoding a 319 amino-acid protein. ${ }^{1}$ It is expressed in the inner cell mass and extraembryonic ectoderm and its expression becomes restricted to neuroectoderm and primordial gem cells at gastrulation. ${ }^{2}$ Soon after, its expression extends to the foregut, neural tube including dorsal root ganglia and primordial germ cells. ${ }^{3}$ Gene-targeting experiments have shown that Sox2 is required very early during embryonic development for the transition of the inner cell mass to the epiblast layer. ${ }^{2}$ At later stages, it plays an essential role for lineage specification determining fate decisions within the three germ layers and in the neuroectoderm, controlling proliferation, morphogenesis and differentiation. ${ }^{4}$ In the mouse, Sox2 regulates germ cell specification at $7.0 \mathrm{dpc}$ germ, while in humans a similar role is played by SOX17, another factor belonging the Sox family. 3 Sox2 heterozygous mutations or deletions lead to congenital disease such as anophthalmia-esophageal-genital syndrome in humans ${ }^{6}$ while a similar condition is generated by Sox2 conditional deletion mice. ${ }^{3}$ SOX2 overexpression, on the contrary, occurs in amplifications of the $3 q 26.3$ gene locus ${ }^{7}$ within esophageal squamous cell carcinoma, glioblastoma, oral squamous cell carcinoma, sinonasal cancer, small cell lung cancer, non-small cell lung cancer ${ }^{7-10}$ and this event has been correlated to neoplastic transformation. In mouse models for squamous carcinomas, Sox2 deletion has been shown to impair skin cancer initiation and development, ${ }^{11}$ whereas its expression cooperates with $L k b 1$ loss in lung carcinomas. ${ }^{12}$ However, it is important to note that SOX2 does not correlate with poor prognosis or decreased progression-free survival in all cancer types, and it has been shown to correlate with a favorable prognosis in lung and a subset of gastric cancers. ${ }^{8,13-15}$ To date, however, its role in controlling melanoma growth and metastasis has not been clearly established.

Melanoma derives from malignant transformation of melanocytes beginning from a benign melanocytic nevus to malignant melanoma via dysplastic nevus. ${ }^{16}$ It accounts for only $4 \%$ of all dermatological cancers but it remains the most lethal by far, accounting for $80 \%$ of all skin cancer-related deaths. ${ }^{17}$ The BRAF gene is the most common somatically mutated gene, occurring in nearly $65 \%$ of all melanoma cases, and the most common mutation is a missense mutation from valine to glutamic acid at amino acid 600 (BRAF ${ }^{\mathrm{V} 600 \mathrm{E}}$ ) in the adenosine triphosphate-binding region of the protein. ${ }^{18}$ Functionally, the BRAFV600E mutation causes a 10-12-fold increase in its activity, triggering the

\footnotetext{
${ }^{1}$ Department of Biomedicine and Prevention, University of Rome Tor Vergata, Rome, Italy; ${ }^{2}$ Regina Elena National Cancer Institute - IFO, Oncogenomic and Epigenetic Unit, Rome, Italy; ${ }^{3}$ Department of Biotechnology and Bioscience, University of Milan-Bicocca, Milan, Italy; ${ }^{4}$ Molecular Oncology Laboratory, Istituto Dermopatico dell'Immacolata - IRCCS,

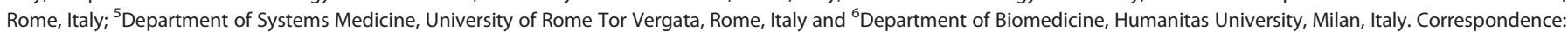
Professor S Dolci, Department of Biomedicine and Prevention, University of Rome Tor Vergata, Via Montpellier 1, 00133 Rome, Italy.

E-mail: dolci@uniroma2.it

${ }^{7}$ These authors contributed equally to this work.

Received 29 November 2016; revised 31 January 2017; accepted 2 February 2017
} 
2

hyperactivation of the mitogen-activated protein kinase signaling cascade leading to cellular survival and proliferation. A link between BRAF ${ }^{\mathrm{V} 600 \mathrm{E}}$ and SOX2 expression has recently investigated in colorectal cancer, suggesting that activation of mitogen- activated protein kinase signaling pathway might induce SOX2 expression. $^{19}$

SOX2 is not expressed in human and mouse melanocytes; however, initial studies showed that SOX2 was highly expressed in

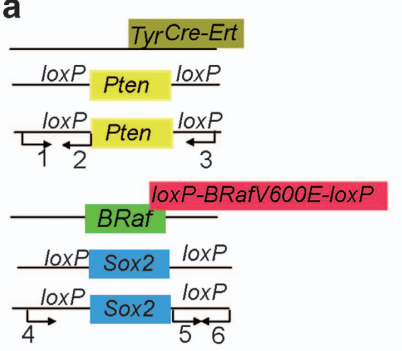

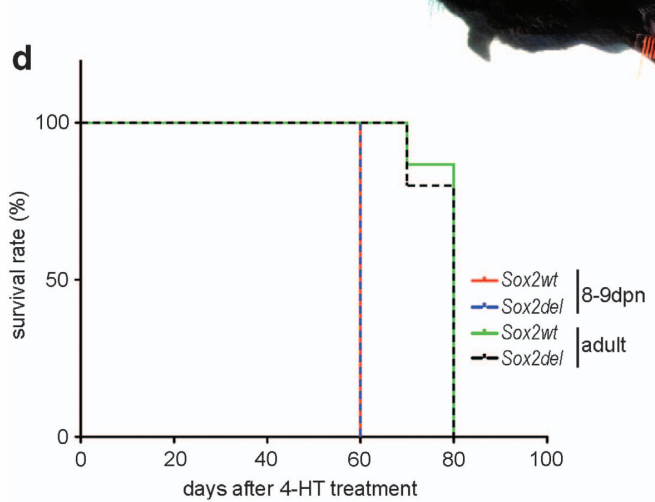

f

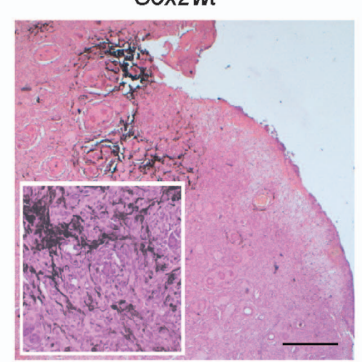

h

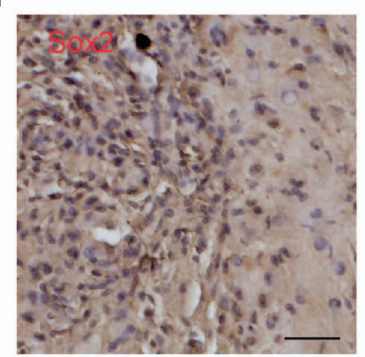

j

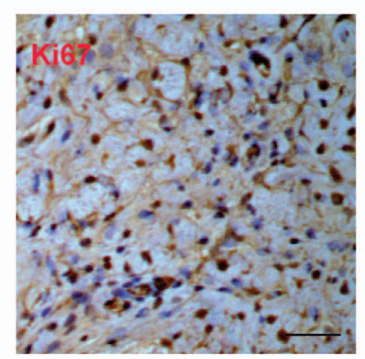

b
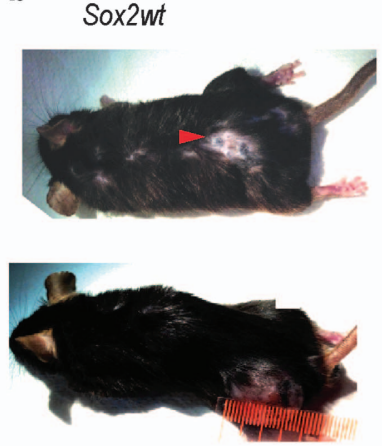
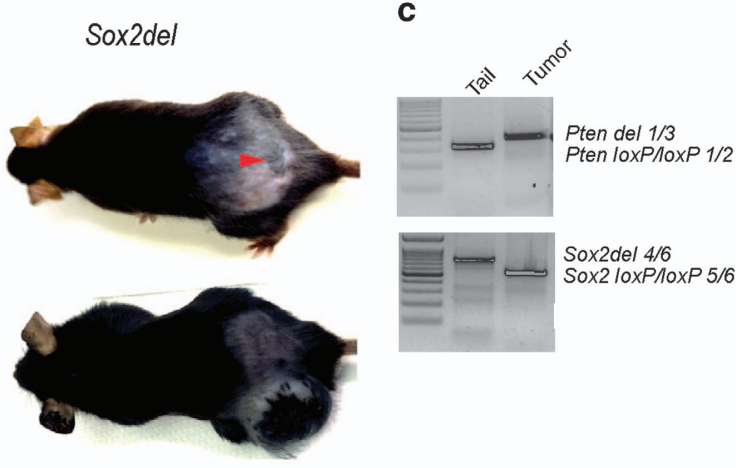

e

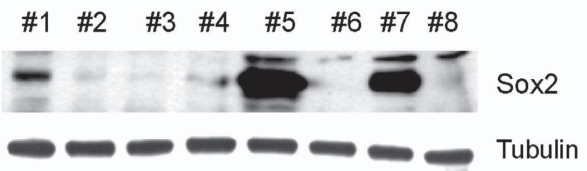

g

Sox $2 w t$

Sox2del
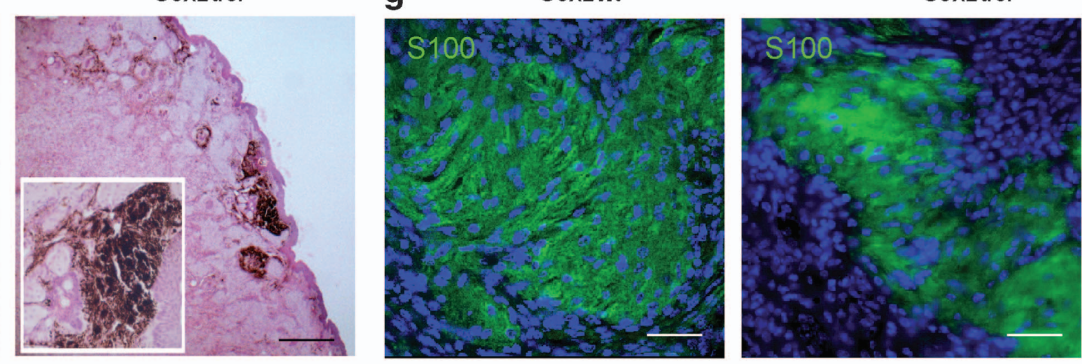

i
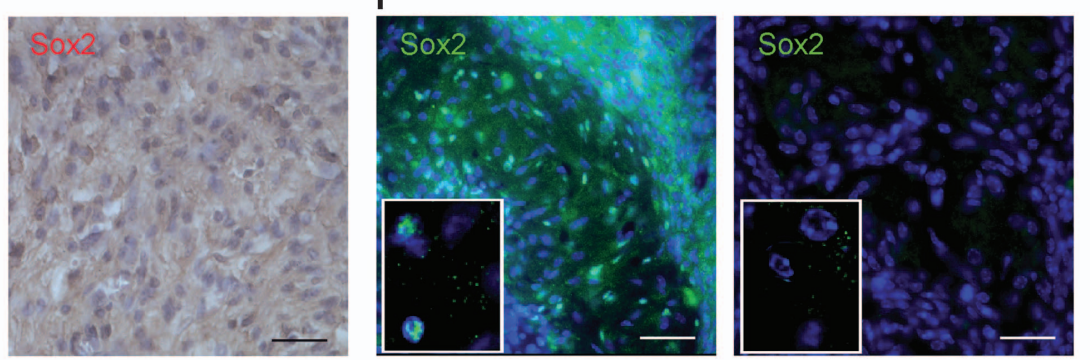

k
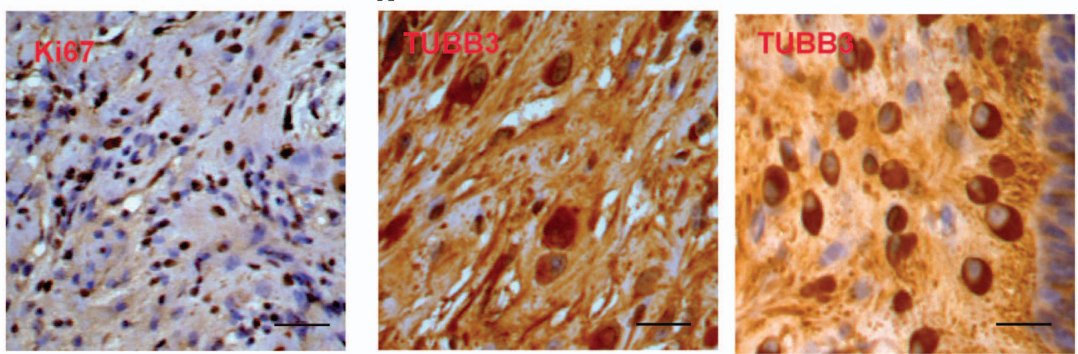
primary melanoma (67\%) and metastatic melanomas (80\%) compared to nevi (14\%). ${ }^{20,21}$ Moreover, SOX2 expression was associated to dermal invasion, which was determined by increased tumor thickness. ${ }^{22}$ More recently, SOX2 expression has been implicated in the regulation of self-renewal and tumorigenicity of melanoma-initiating cells. ${ }^{23}$

To address the role in melanoma development, we employed the Cre-loxP strategy to delete Sox2 exon specifically in melanocytes in a BRaf ${ }^{\mathrm{V} 600 \mathrm{E}}$ and Pten null melanoma mouse model. ${ }^{24}$

Our results show that Sox2 is not essential for melanoma development, nor for melanoma growth and metastasis. Bioinformatic analysis showed that Sox2 levels were not significantly different between benign nevi and melanomas, moreover analysis on two melanoma patient cohorts confirmed that Sox2 levels did not significantly change between primary and metastatic melanoma. We also found that Sox 2 expression was not required to acquire vemurafenib resistance both in mouse and in human V600E BRAF-mutated cell lines. Altogether, our results suggest that Sox2 is dispensable for melanoma development and that its expression does not correlate to BRAF inhibitor (BRAFi) treatment resistance.

\section{RESULTS AND DISCUSSION}

Sox2 deletion does not influence melanoma development and metastasis formation in BRaf ${ }^{V 600 E} ;$ Pten $^{-/-}$; Tyr::Cre-ER mice

It has been recently shown that SOX2 knockdown affects human melanoma xenografts, self-renewal of human melanoma spheres and putative melanoma stem cells self-renewal in vitro. ${ }^{23}$ To assess the role of Sox 2 in a mouse melanoma model, we crossed mice carrying Sox2 floxed alleles $\left(\text { Sox } 2^{\text {loxP/loxP }}\right)^{25}$ with Pten ${ }^{\text {loxP/loxP }}$ $B R a f^{V 600 E}$ Tyr:: CreER (carrying a transgene in which the expression of Cre recombinase fused to the tamoxifen-binding domain is driven by the tyrosinase promoter ${ }^{26}$ ) mice. ${ }^{24}$ This model harbors the BRaf ${ }^{V 600 E}$ mutation and Pten deletion upon 4-hydroxytamoxifen (4-HT) treatment by local cutaneous administration. Treated mice develop melanomas, which recapitulate key pathophysiological aspects of the human BRAF-activated and PTEN null-driven melanoma, including lymph node and lung metastasis, with $100 \%$ penetrance and short latency. ${ }^{24}$ We set up matings with Sox2 IoxPloxp mice to obtain experimental animals Sox ${ }^{\text {wt } / w t}$; Pten ${ }^{\text {loxp/loxp }} ;$ BRaf $^{\text {V600E }}$; Tyr::CreER (Sox2wt) and Sox $2^{\text {loxP/loxP }}$; Pten ${ }^{\text {loxP'loxP }}$; BRaf ${ }^{V 600 E}$ Tyr::CreER (Sox2del; see Figure 1a). Fifteen adult (7-8 weeks) and five prepuberal (8-9 days post natum) individuals per group were topically treated with 4-HT. We then monitored the development and progression of melanoma in the presence and absence of Sox2, by assessing hyper-pigmentation, tumor occurrence and growth differences between the two groups. Similarly to the control Sox2wt group, the Sox2del mice showed melanocyte-specific expression of Braf ${ }^{\mathrm{V} 600 \mathrm{E}}$ as revealed by hyper-pigmentation at around 20 days following local administration of 4-HT (Figure 1b). We found that adult mice both from Sox2wt and Sox2del developed tumors around 45 days after 4-HT treatment and mice required euthanasia 70-80 days following 4HT administration when the tumor mass reached $2 \mathrm{~cm}$ diameter (Figure 1b). The timing of hyper-pigmentation and melanoma occurrence was accelerated in prepuberal mice, and nevi appeared at around 15 days after 4-HT treatment, while melanoma nodules were visible at 40 days and required killing at 50-60 days after 4-HT local treatment, however no differences were found between Sox2wt and Sox2del mice (Figure 1d). Deletion of Sox 2 was confirmed by PCR analysis on genomic DNA obtained from tumor lesions (Figure 1c), by western blot analysis on tumor extracts (Figure 1e) and immunofluorescence on primary lesions derived from 4-HT-treated Sox2del mice. We found no gross histological differences between tumors expressing Sox 2 and those carrying the deleted alleles (Figure 1f). Lesions from both genotypes showed variable pigmentation on the surface of the melanoma, whereas in the deeper layers densely packed amelanotic tumor cells were found. Within the affected areas we observed melanoma aggregates with nerve sheath-like

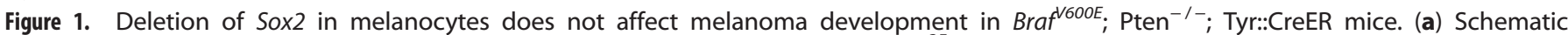
representation of the conditional knockout/knockin genotypes, in which both Sox $2^{25}$ and Pten alleles are flanked by loxP sequences and one of the two BRaf allele contains an exon 15-18 cassette flanked by two loxP sites that precedes the mutated exon 15 (V600E). ${ }^{24,43}$ Tyr-Cre-Ert2 transgene drives the expression of Cre specifically in melanocytes. ${ }^{26}$ Pten ${ }^{\text {loxP/loxP }}$; BRaf ${ }^{V 600 E}$; Tyr::Cre-Ert2 mice were obtained from Jackson Laboratories (Bar Harbor, ME, USA). Arrows and numbers indicate PCR primers for detection of floxed alleles or Cre-mediated recombination bands following local treatment with 4-HT (see c). All animal experiments were approved by the University of Rome Torvergata Ethics Committee for Animal Experiments. (b) Representative picture of Sox2wt and Sox2del mice after 15-20 days (upper panels, red arrowheads indicate hyper-pigmentation) and 70 days (lower panels, melanomas) from local 4-HT administration. 1.5 $\mu$ l of a 4-HT solution (5 mM in ethanol, Sigma-Aldrich, Milan, Italy) was applied with a paintbrush onto the dorsal skin of 7-8 week-old mice. No randomization and blinding was used in animal experiments. (c) Cre-mediated recombination of Pten and Sox2 loci revealed by PCR amplification of deletion bands on tumor or tail DNA using the indicated primers (for Pten primers, see Dankort et al.; ${ }^{43}$ Sox2 primer 4 for: 5'-CATTGGAGAGGTTCAGACTA-3';

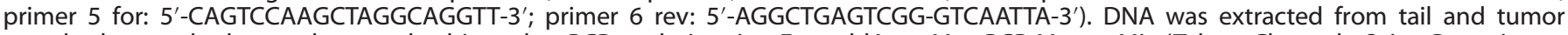
samples by standard procedures and subjected to PCR analysis using EmeraldAmp Max PCR Master Mix (Takara-Clontech, Saint-Germain-enLaye, FR). (d) Kaplan-Meier survival curves for Sox2wt and $(n=15+5)$ and Sox2del $(n=15+5)$ mice following local administration of $4-H T$. Mice from 10 different litters were analyzed. Survival curves were plotted using the Kaplan-Meier method and differences between groups were evaluated using the log-rank test. No statistically significant differences were found between the two groups $(P=0.63$ for adult mice and $P=1$ for prepuberal mice). (e) Western blot analysis for Sox2 expression on melanoma extracts from different genotypes. \#1: Sox ${ }^{\text {wt/loxP }}$; \#2, \#3, \#4, \#6 and \#8: Sox $2^{\text {loxP/loxp }}$, \#5 and \#7: Sox $2^{+/+}$. (f) Hematoxylin-eosin (H\&E) staining and (g) S100 immunofluorescence on tumors from Sox2wt and Sox2del mice following local 4-HT administration. Paraffin sections $(4 \mu \mathrm{m})$ were stained with H\&E solution (Dako, Glostrup, Denmark) or retrieved with citrate buffer solution and probed with rabbit anti-S100 antibodies (Z0311, Dako, 1:100) followed by incubation with

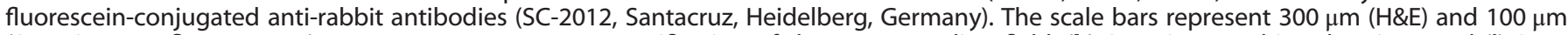
(S100 immunofluorescence). Insets represent a $\times 10$ magnification of the corresponding field. (h) Sox2 immunohistochemistry and (i) Sox2 immunofluorescence on tumors from Sox2wt and Sox2del mice following local 4-HT administration. (j) Ki-67 immunohistochemistry on Sox2wt

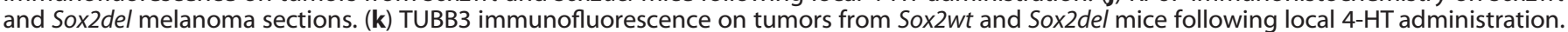
The scale bars represent $100 \mu \mathrm{m}$. Insets represent $\times 3$ magnification of the corresponding field. Immunohistochemistry was performed on paraffin sections, retrieved in EDTA solution (Dako) and probed with rabbit anti-Ki-67 antibodies (sc-15402, SantaCruz, 1:100), with mouse or anti-rabbit anti-Sox2 antibodies (AB56031:100, Millipore, Milan, Italy) or with mouse anti Tubulin beta3 antibodies (CBL270 EMD Millipore) followed by avidin-biotin-peroxidase antibodies or by fluorescein-conjugated anti-rabbit antibodies. The percentage of Ki-67-labeled nuclei was obtained by counting Ki-67-positive nuclei out of 1000 tumor cell nuclei within comparable section areas between the two genotypes at a $\times 20$ magnification (counts were obtained from three different experiments, data represent the mean \pm s.d.). Mayer hematoxylin (Dako) or Hoechst 33349 (Sigma-Aldrich) were used as nuclear counterstains. Deconvolution and microscopy inspections were performed on a Leica CTR6000 microscope. 
a

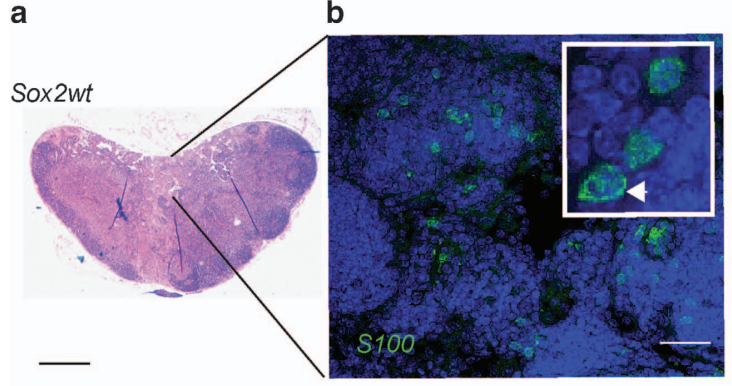

Lymph Node

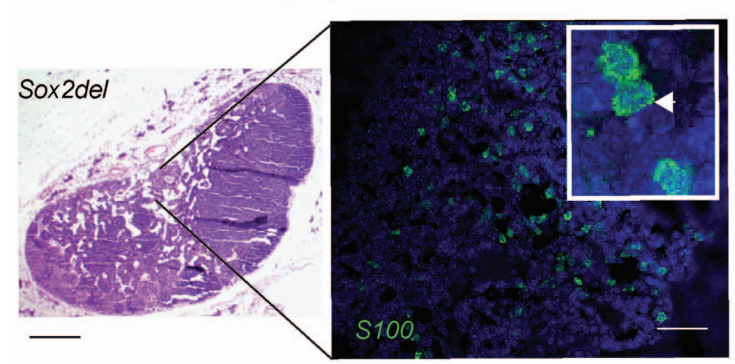

c

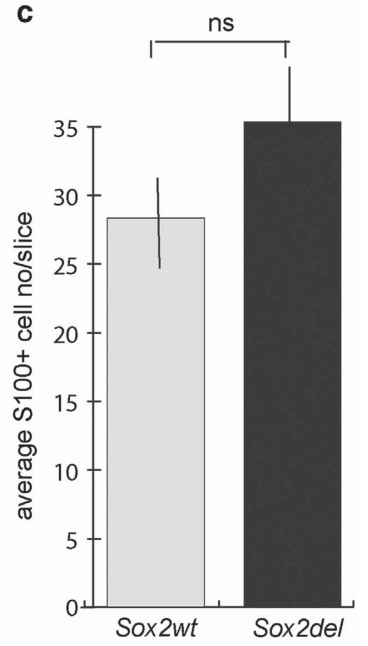

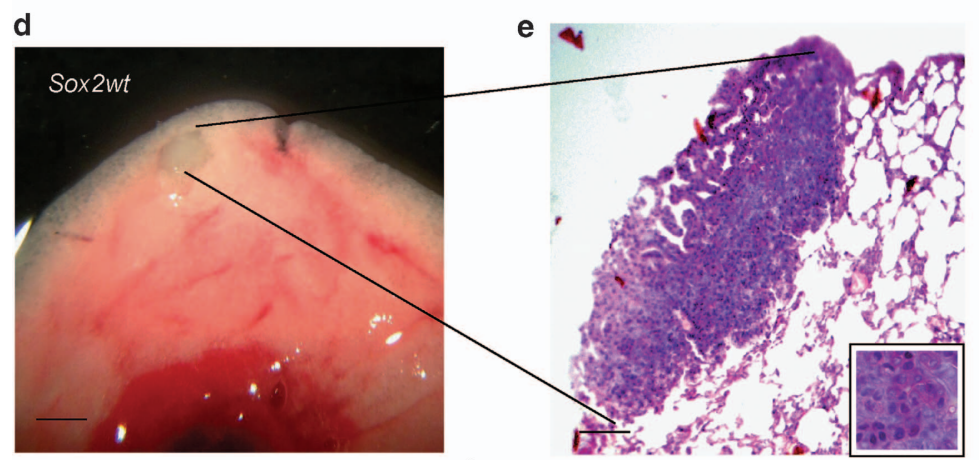

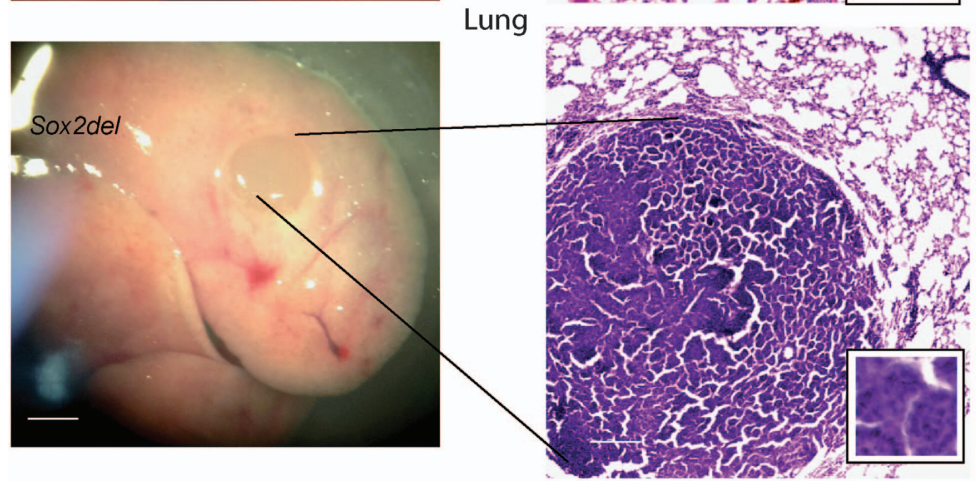

Figure 2. Deletion of Sox2 in melanocytes does not affect melanoma lymph node and lung metastases. (a) H\&E staining and (b) S100 immunostaining of lymph node sections from Sox2wt (upper panel) and Sox2del (bottom panel) mice following local 4-HT administration. Scale bars represent $300 \mu \mathrm{m}$ (H\&E) and $100 \mu \mathrm{m}$ (IF). Insets represent $\times 5$ magnification of the corresponding field. (c) Quantification of metastasis in lymph nodes of Sox2wt (left) and Sox2del mice. To quantify the number of melanoma cells infiltrating the lymph nodes, each other section (four sections) from three different lymph node sections were S100 immunostained. S100-positive cells within lymph node sections with comparable areas between the two genotypes were counted at a $\times 20$ magnification $(n=351$, Sox $2 w t ; n=402$, Sox 2 del). The data represent the mean \pm s.d. of cell numbers/sectioned area ( $n=3$ mice, $P=0.14$, unpaired $t$ test, Prism 5 (Graphpad) software). (d) Stereomicroscopic inspection of affected lung (scale bar $=500 \mu \mathrm{m})$. Black lines point to peripheral metastases that were histologically analyzed by H\&E. Scale bars represent $100 \mu \mathrm{m}$. Insets represent a $\times 5$ magnification of the corresponding field. (e) Quantification of lung metastases was performed by counting the number of nodular lesions within the surface of the lungs. Nodules were amelanotic.

morphology (Figure 1g), similar to what has been described in Pten/BRaf/ $\beta$ catenin KO mice. ${ }^{27}$ These cells showed vesicular nuclei containing small clumps of condensed chromatin and abundant cytoplasm. The same features were found in the tumors obtained in prepuberal mice (not shown). Tumors collected from treated mice were further characterized by using the melanoma marker $\mathrm{S} 100$. We found that lesions from both genotypes positively stained for S100, while, as expected, only Sox2wt were positive for Sox2 labeling (Figures 1g-i). Although melanomas showed similar growth kinetics, as judged by tumor diameter, we also found that 
a

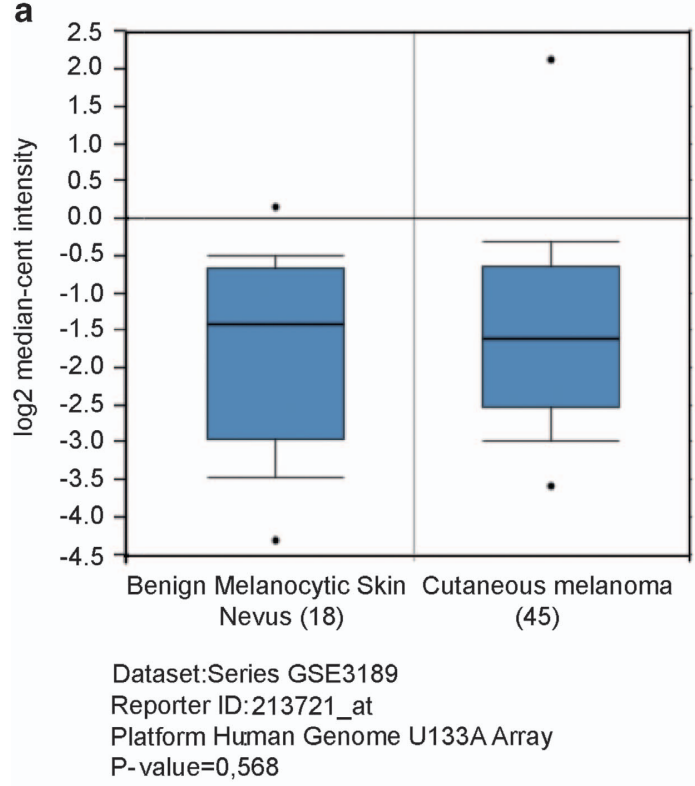

b

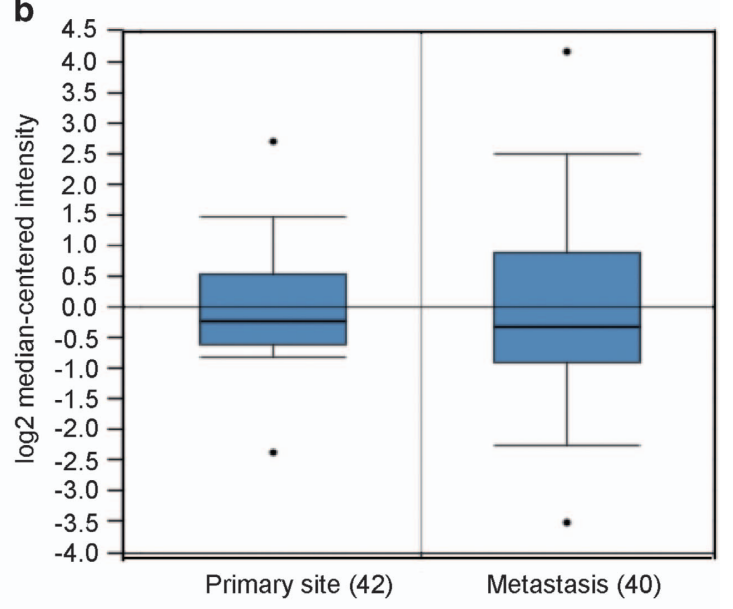

Dataset: Series GSE7553

Reporter ID: 213721_at

Platform: Human Genome U133 Plus 2.0 Array P-value $=0,732$

C

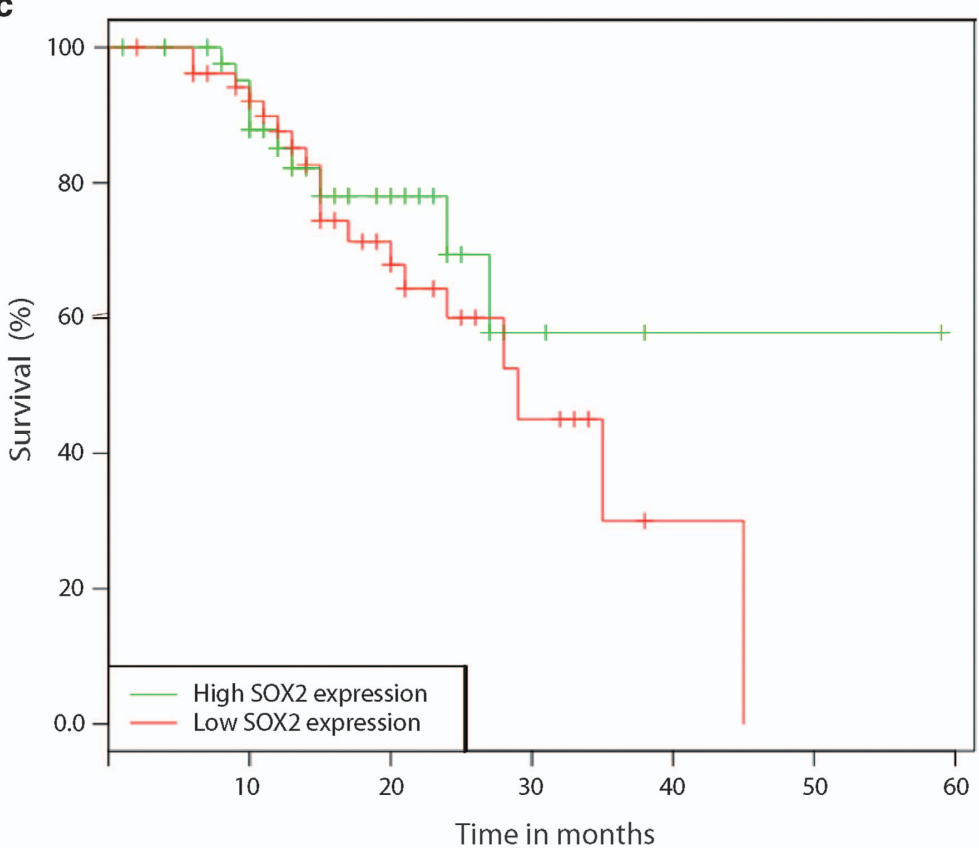

GEO dataset ID: TCGA SKCM

(Skin Cutaneous Melanoma)

Cancer Type: Skin

Clinical Variable: ALL

Number of samples: 103

Statistical details

Submitted Gene: SOX2

probe ID: SOX2

p-value: 0.532

Figure 3. SOX2 expression in human melanoma does not correlate with tumor aggressiveness and patient survival. (a-b) Box plots represent relative SOX2 mRNA expression levels in malignant melanoma $(n=45)$ and benign melanocytic lesions $(n=18)($ Talantov data set; GSE3189) and in primary $(n=42)$ and metastatic melanoma $(n=40)$ (Riker data set; GSE7553). Bars represent \pm s.d. Data processing was performed using BioConductor packages (http://www.bioconductor.org/) in the R Computing Environment version 3.0.2 (http://www.r-project.org/). A value of $P<0.05$ was considered statistically significant. (c) Kaplan-Meier survival curve of SOX2 expression in skin cutaneous melanoma (TCGA_SKCM). Melanoma cancer patients with high $(n=45)$ and low $(n=53)$ Sox2 expression had a comparable 5-year survival $(P=0.532)$. Survival curves were compared between groups using log-rank test. Statistical analyses were performed by ANOVA analysis using R project tool. A value of $P<0.05$ was considered statistically significant.

the percentage of Ki67-positive cells was not different between the two genotypes $(32 \pm 2 \%$ in Sox $2 w t$ vs $34 \pm 4 \%$ in Sox2del, $P=0.65$, unpaired $t$-test) indicating that Sox 2 is not essential for melanoma proliferation (Figure 1j). Human skin melanocytes and most primary malignant melanomas are TUBULIN- $\beta$ III (TUBB3) positive, ${ }^{28}$ and loss of TUBB3 protein correlates with chemosensitivity to microtubule-interfering drugs in malignant melanoma cells. We found that also mouse melanoma cells express high levels of Tubb3, however its expression was not regulated by Sox2 deletion (Figure $1 \mathrm{k}$ ).

We then examined the possible role of Sox2 expression to regulate the ability of melanoma cancer cells to metastasize to lymph nodes and lungs. We found that inguinal lymph nodes adjacent to the primary lesions were always affected by melanoma metastasis either in Sox2wt or in Sox2del mice (Figures 2a and b). By immunofluorescence analysis for the melanoma marker S100, we 
evaluated the amount of positive cells within the Sox2wt- or Sox2del-affected lymph nodes (Figure $2 \mathrm{~b}$ ). We found that the number of S100 infiltrating cells were similar between the two genotypes, suggesting that Sox2 deletion does not affect the ability to colonize the lymph nodes (Figure 2c). We found that both genotypes were also affected by 1 or 2 lung macro-metastases (Figures $2 \mathrm{~d}$ and e) but we did not observe differences in number, as evaluated at microscope inspection. Histology confirmed that the lung lesions consisted of densely packed cells with a cord-like architecture that completely disrupted the lung alveolar structure
(Figure 2c). Altogether these results demonstrate that Sox2 is not required for melanomagenesis in $B R a f^{V 600 E}$ Pten null ${ }^{29}$ mouse models, nor for melanoma growth and metastasis.

Sox2 expression is not differentially expressed during human melanoma onset and metastasis

Next, we wondered if a relationship existed between SOX2 mRNA levels and melanoma disease. To answer this, we conducted a meta-analysis in a public melanoma cancer data set derived from
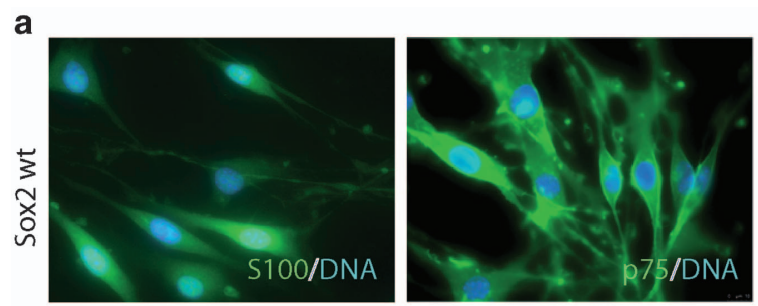

b
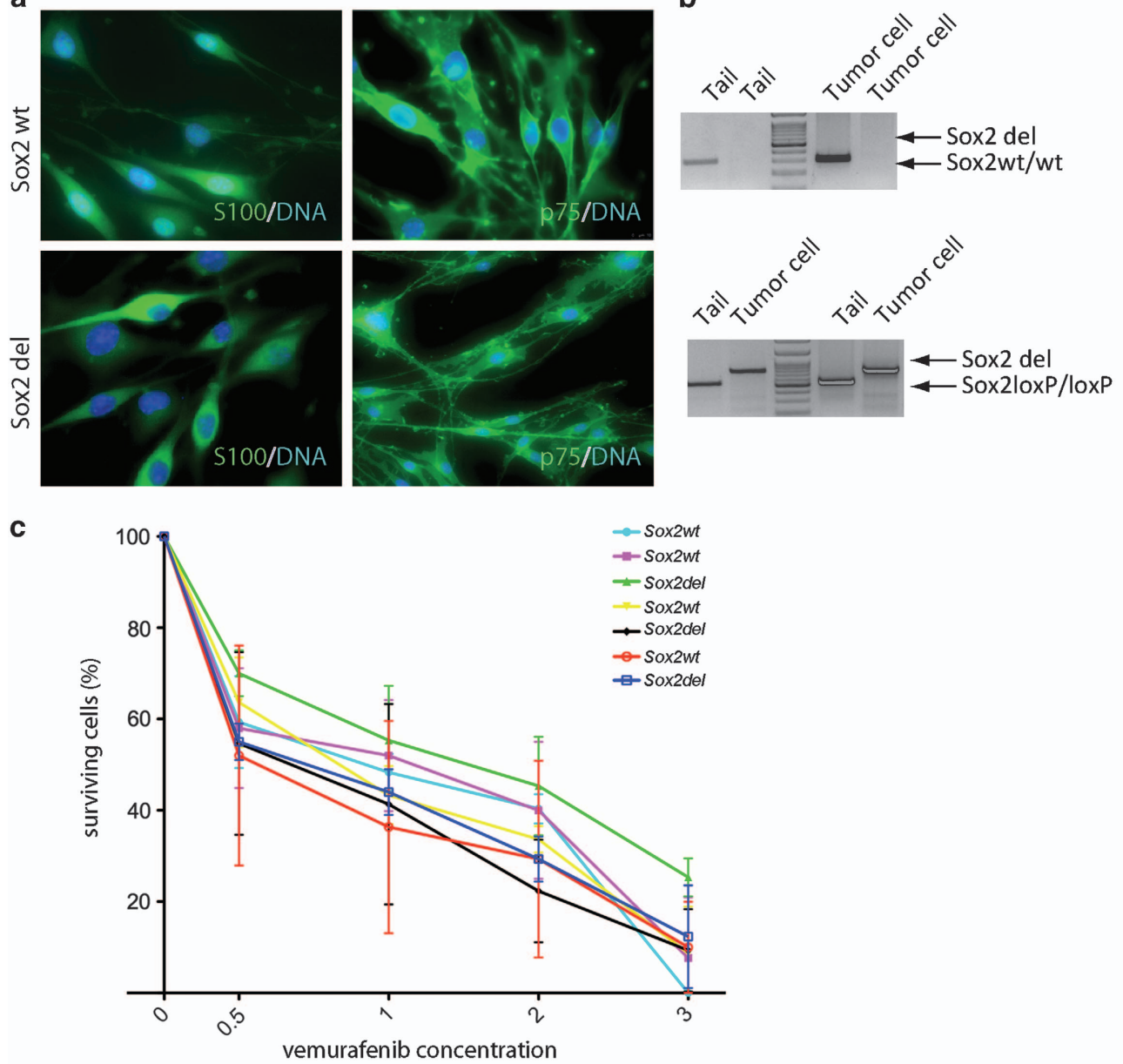

d

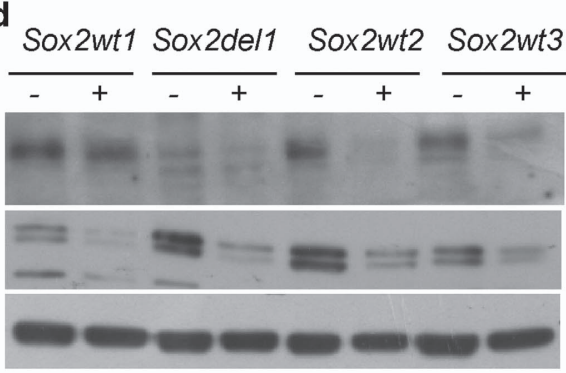

Sox2wt4 Sox $2 w t 5$ Sox 2 del 2

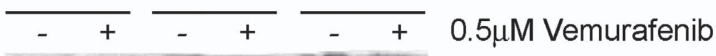

e
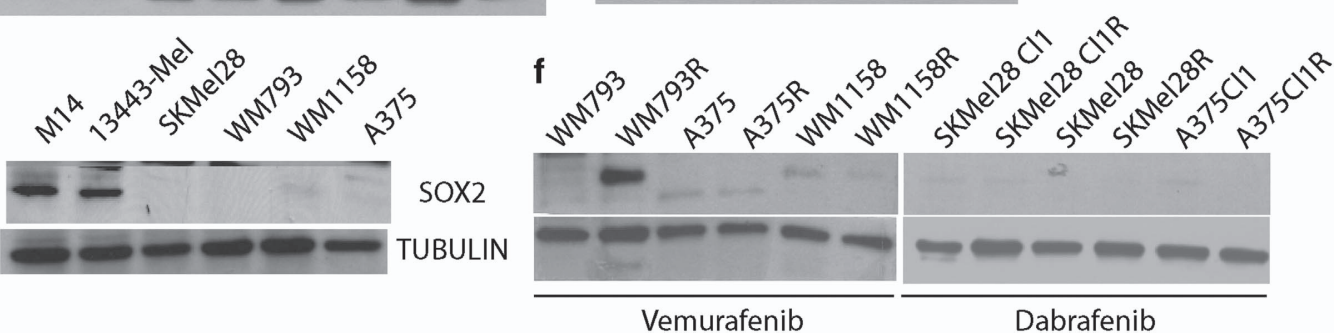
Figure 4. Sox2 deletion does not affect melanoma sensitivity and is not correlated to resistance acquisition to BRAFi treatments. (a) S100 and p75 (rabbit anti-p75 AB8874, Abcam, Cambridge, UK) immunofluorescence on melanoma cell lines and (b) PCR genotypes to detect the deletion band corresponding to the fragment generated from the deleted Sox2 locus, from the floxed or wt alleles. (c) IC 50 curve on Sox2wt or Sox2del mouse melanoma cell lines. Cells were incubated in the presence or absence of increasing concentration of vemurafenib $(\mu \mathrm{m}$ Genentech, San Francisco, CA, USA) dissolved at $10 \mathrm{~mm}$ concentration in dimethylsulphoxide and diluted at the corresponding concentrations in culture medium. Cells were cultured in Dulbecco Modified Eagle Medium 10\% fetal bovine serum (Lonza, Milan, Italy), seeded in 96 multi-wells (Falcon, Milan, Italy) and incubated for $120 \mathrm{~h}$ before performing 3-(4,5-dimethylthiazol-2-yl)-5-(3-carboxymethoxyphenyl)-2-(4-sulfophenyl)-2H-tetrazolium, inner salt assay (Promega, Rome, Italy). Experiments were performed in triplicate and repeated three times. Bars represent \pm s.d. Statistical analysis was done by Kruskal-Wallis test $(P=0.97$; Prism5 software). (d) Sox2, pErks and Erk2 expression by western blot analysis in Sox $2 w t$ and Sox 2 del cell lines after $72 \mathrm{~h}$ of incubation with $0.5 \mu \mathrm{m}$ vemurafenib. Blots are representative of four independent experiments. (e) SOX2 expression by western blot analysis on human melanoma cell lines. (f) SOX2 expression by western blot analysis on human melanoma cell lines sensitive or resistant to BRAFi treatment. Blots are representative of three independent experiments. The human cell lines used in this work have been described in Graziani et al., ${ }^{33}$ Lacal et al. ${ }^{34} \mathrm{M} 14$ cell line was a generous gift from Dr G Zupi (Regina Elena Cancer Institute, Rome, Italy); 13443-Mel cell line was a generous gift from Dr G Parmiani (Istituto Nazionale Tumori, Milan, Italy); WM1158, SK-Mel28, WM793 and A375 cell lines were purchased from the American Type Culture Collection (Manassas, VA, USA). Cells were authenticated (Genetica DNA Lab. Inc., Cincinnati, OH, USA) and routinely tested for mycoplasma contamination. Cell were cultured in RPMI 10\% fetal bovine serum (Lonza). The acquisition of BRAFi resistance was generated in our laboratories by exposing the parental cell line to increasing concentrations of vemurafenib or dabrafenib for 3 months. Cells were treated with $3 \mu \mathrm{m}$ vemurafenib as for mouse cells or with $1.5 \mu \mathrm{m}$ dabrafenib (GSK2118436A, dissolved at $1.92 \mathrm{~mm}$ in dimethylsulphoxide). In all the experiments, the variation within each experimental group was estimated to ensure that the variance was similar for groups that were being statistically compared.

gene expression microarray analysis on clinical samples (Figure 3a). We did not observe a significant correlation of SOX2 expression with malignant melanoma or benign melanocytic lesions $(P=0.568)$. Interestingly, SOX2 expression did not change between primary tumors and metastatic melanoma tissues $(P=0.732$; Figure $3 \mathrm{~b})$. To evaluate the prognostic value of SOX2 expression in melanoma patients, 5-year overall survival rate was analyzed through the Kaplan-Meier plot in a TCGA data set (Figure 3c). The analysis revealed that SOX2 mRNA levels did not affect the survival of patients $(P=0.532)$, supporting the previous evidences that its high or low expression was not associated with melanoma cancer. ${ }^{29,30}$ Although SOX2 mRNA levels may not completely reflect protein expression levels, these results suggest that SOX2 expression does not correlate with melanoma onset, growth and metastatic ability and it cannot be considered a prognostic factor.

Sensitivity and acquisition of resistance to BRAFi of human and mouse melanoma cell lines do not depend upon SOX2 expression We next investigated if Sox2wt and Sox2del melanoma cells could differentially respond to BRAFi treatments. To this end, we established melanoma cell lines from the two Sox2 genotypes and confirmed that they were melanoma cells by S100 and p75NGFR immunostaining and by PCR for Pten deletion and Sox2 wt/deletion bands (Figures $4 \mathrm{a}$ and $\mathrm{b}$ ). The response of melanoma cells to the BRAFi vemurafenib was evaluated using the $50 \%$ inhibitory concentration $\left(\mathrm{IC}_{50}\right)$ assay calculated after 5 days of culture by 3-(4,5-dimethylthiazol-2-yl)-5-(3-carboxymethoxyphenyl)-2-(4-sulfophenyl)-2H-tetrazolium, inner salt (Figure 4c). We found a clustering of $\mathrm{IC}_{50}$ values at $500 \mathrm{~nm}$ for the all the cell lines regardless they were Sox2wt or Sox2del and most cell lines were completely halted at $3 \mu \mathrm{m}$ vemurafenib. It has been reported that mitogen-activated protein kinase inhibition does not influence SOX2 levels in human lung stem cells, ${ }^{31}$ however by western blot analysis, we found that Sox $2 w t$ cells treated with $500 \mathrm{~nm}$ vemurafenib, downregulated Sox2 and Erk phosphorylation (Figure $4 d$ ), similarly to what has been shown in colon cancer. ${ }^{32}$ This effect suggests that Sox 2 expression can be induced by the BRaf-activated pathway in BRaf ${ }^{\mathrm{V} 600}$-mutated melanocytes, however it is dispensable for melanocyte transformation. When we compared the ability to develop vemurafenib resistance, we found that Sox2wt and Sox2del cells behaved similarly when cultured in the presence of $3 \mu \mathrm{m}$ vemurafenib concentration in long-term cultures (not shown), further supporting the lack of a role of Sox2 in the acquisition of BRAFi resistance. We also investigated if any differences in the acquisition of resistance to BRAFi treatments could be evaluated in SOX2 expressing and non-expressing human melanoma cell lines. We first assessed SOX2 expression in cell lines that were previously identified as vemurafenib- or dabrafenib-sensitive according to their $\mathrm{IC}_{50}$ (G Graziani personal communication and; ${ }^{33,34}$ Figure $\left.4 \mathrm{e}\right)$. We then set up long-term cultures of SOX2-expressing or SOX2-negative cells in the presence of $3 \mu \mathrm{m}$ vemurafenib or $1.5 \mu \mathrm{m}$ dabrafenib. As shown in Figure $4 \mathrm{f}$, we found that both SOX2-positive and SOX2-negative cells reached vemurafenib or dabrafenib resistance independently from SOX2 expression. Notably, we found that only in one out of four SOX2 non-expressing cell lines, SOX2 levels were upregulated, while in one cell line expressing low SOX2 levels vemurafenib almost completely abolished SOX2 expression. Altogether, these results show that SOX2 expression is dispensable either for sensitivity or for the acquisition of resistance to BRAFi treatments. Since acquisition of BRAFi resistance correlates with an increase of invasiveness of melanoma cells, ${ }^{35}$ our results further support the evidence that SOX2 expression does not correlate to an invasive phenotype.

SOX2 deregulation has been involved in the oncogenetic process of several cancer types, mainly due to its association with cancer stem cells. ${ }^{36}$ The tumor origin and cellular context, however, have been shown to control the outcome of SOX2 activation, probably reflecting its role during development. In the majority of cases, SOX2 can induce aberrant cell growth and tumorigenesis, while genetic inactivation can revert self-renewal and tumor growth. Controversial is its role in gastric cancer, being correlated to decreased cancer aggressiveness and better patient outcome by one group ${ }^{15}$ or involved in increased metastasis and chemo-resistance by other authors. ${ }^{37,38}$

Our results do not support an essential role of SOX2 in the process of melanomagenesis, nor in melanoma growth, metastasis, patient outcome and in vitro chemo-sensitivity to BRAFi therapy. Our evidences are based on several data: first, this paper and the accompanying paper by Schaefer et al. ${ }^{39}$ present two independent mouse models in which Sox2 deletion in melanocytes undergoing cell transformation does not prevent melanoma growth and metastasis. Second, analysis on publicly available databases did not reveal any significant differences of SOX2 mRNA levels in benign melanocytic lesions, such as nevi, primary and metastatic melanomas, and showed that its expression is not a prognostic factor for patient survival. Finally, we do not find differences in the response to BRAFi treatments of Sox2wt and Sox2del cells in vitro. While expression of Sox superfamily members can overlap during development ${ }^{40,41}$ they do not 
substitute Sox 2 loss of functions, it is possible that expression of at least one of these members, such as Sox10, can support melanoma development ${ }^{42}$ in the absence of Sox 2 during the process of melanomagenesis. This hypothesis, however, excludes that Sox2 plays an essential role in melanocyte transformation and melanoma growth.

\section{CONFLICT OF INTEREST}

The authors declare no conflict of interest.

\section{ACKNOWLEDGEMENTS}

We thank $G$ Graziani for helpful criticisms and for sharing results with us and $G$ Rossi for histology assistance. We wish to thank Genentek (San Francisco, CA, USA) for the supply of vemurafenib and GlaxoSmithKline (Brentford, UK) for the supply of dabrafenib. SN is supported by Telethon and AIRC grants. SC is recipient of a fellowship from AIRC (Investigator Grant Project 17585). This work has been supported by MIUR Grants 2015XSNA83_006 and 2015XCR88M.

\section{REFERENCES}

1 Kamachi $\mathrm{Y}$, Uchikawa $\mathrm{M}$, Kondoh $\mathrm{H}$. Pairing SOX off: with partners in the regulation of embryonic development. Trends Genet 2000; 16: 182-187.

2 Avilion AA, Nicolis SK, Pevny LH, Perez L, Vivian N, Lovell-Badge R. Multipotent cell lineages in early mouse development depend on SOX2 function. Genes Dev 2003; 17: $126-140$.

3 Campolo F, Gori M, Favaro R, Nicolis S, Pellegrini M, Botti F et al. Essential role of Sox2 for the establishment and maintenance of the germ cell line. Stem Cells 2013; 31: 1408-1421.

4 Sarkar A, Hochedlinger K. The sox family of transcription factors: versatile regulators of stem and progenitor cell fate. Cell Stem Cell 2013; 12: 15-30.

5 Irie N, Weinberger L, Tang WW, Kobayashi T, Viukov S, Manor YS et al. SOX17 is a critical specifier of human primordial germ cell fate. Cell 2015; 160: 253-268.

6 Wegner M. From head to toes: the multiple facets of Sox proteins. Nucleic Acids Res 1999; 27: 1409-1420.

7 Hussenet T, Dali S, Exinger J, Monga B, Jost B, Dembele D et al. SOX2 is an oncogene activated by recurrent $3 q 26.3$ amplifications in human lung squamous cell carcinomas. PLoS One 2010; 5: e8960.

8 Toschi L, Finocchiaro G, Nguyen TT, Skokan MC, Giordano L, Gianoncelli L et al. Increased SOX2 gene copy number is associated with FGFR1 and PIK3CA gene gain in non-small cell lung cancer and predicts improved survival in early stage disease. PLoS One 2014; 9: e95303.

9 Rudin CM, Durinck S, Stawiski EW, Poirier JT, Modrusan Z, Shames DS et al. Comprehensive genomic analysis identifies SOX2 as a frequently amplified gene in small-cell lung cancer. Nat Genet 2012; 44: 1111-1116.

10 Bass AJ, Watanabe H, Mermel CH, Yu S, Perner S, Verhaak RG et al. SOX2 is an amplified lineage-survival oncogene in lung and esophageal squamous cell carcinomas. Nat Genet 2009; 41: 1238-1242.

11 Boumahdi S, Driessens G, Lapouge G, Rorive S, Nassar D, Le Mercier M et al. SOX2 controls tumour initiation and cancer stem-cell functions in squamous-cell carcinoma. Nature 2014; 511: 246-250.

12 Mukhopadhyay A, Berrett KC, Kc U, Clair PM, Pop SM, Carr SR et al. Sox2 cooperates with Lkb1 loss in a mouse model of squamous cell lung cancer. Cell Rep 2014; 8: 40-49.

13 Wilbertz T, Wagner P, Petersen K, Stiedl AC, Scheble VJ, Maier S et al. SOX2 gene amplification and protein overexpression are associated with better outcome in squamous cell lung cancer. Mod Pathol 2011; 24: 944-953.

14 Chen Y, Huang Y, Chen J, Wang S, Zhou J. The prognostic value of SOX2 expression in non-small cell lung cancer: a meta-analysis. PLoS One 2013; 8: e71140.

15 Otsubo T, Akiyama Y, Yanagihara K, Yuasa Y. SOX2 is frequently downregulated in gastric cancers and inhibits cell growth through cell-cycle arrest and apoptosis. $\mathrm{Br}$ J Cancer 2008; 98: 824-831.

16 Tsao H, Chin L, Garraway LA, Fisher DE. Melanoma: from mutations to medicine. Genes Dev 2012; 26: 1131-1155.

17 Miller AJ, Mihm MC Jr. Melanoma. N Engl J Med 2006; 355: 51-65.

18 Shinozaki M, Fujimoto A, Morton DL, Hoon DS. Incidence of BRAF oncogene mutation and clinical relevance for primary cutaneous melanomas. Clin Cancer Res 2004; 10: 1753-1757.

19 Lundberg IV, Lofgren Burstrom A, Edin S, Eklof V, Oberg A, Stenling R et al. SOX2 expression is regulated by BRAF and contributes to poor patient prognosis in colorectal cancer. PLoS One 2014; 9: e101957.
20 Laga AC, Zhan Q, Weishaupt C, Ma J, Frank MH, Murphy GF. SOX2 and nestin expression in human melanoma: an immunohistochemical and experimental study. Exp Dermatol 2011; 20: 339-345.

21 Laga AC, Lai CY, Zhan Q, Huang SJ, Velazquez EF, Yang Q et al. Expression of the embryonic stem cell transcription factor SOX2 in human skin: relevance to melanocyte and merkel cell biology. Am J Pathol 2010; 176: 903-913.

22 Girouard SD, Laga AC, Mihm MC, Scolyer RA, Thompson JF, Zhan Q et al. SOX2 contributes to melanoma cell invasion. Lab Invest 2012; 92: 362-370.

23 Santini R, Pietrobono S, Pandolfi S, Montagnani V, D'Amico M, Penachioni JY et al. SOX2 regulates self-renewal and tumorigenicity of human melanoma-initiating cells. Oncogene 2014; 33: 4697-4708.

24 Dankort D, Curley DP, Cartlidge RA, Nelson B, Karnezis AN, Damsky WE Jr et al. Braf(V600E) cooperates with Pten loss to induce metastatic melanoma. Nat Genet 2009; 41: 544-552.

25 Favaro R, Valotta M, Ferri AL, Latorre E, Mariani J, Giachino C et al. Hippocampal development and neural stem cell maintenance require Sox2-dependent regulation of Shh. Nat Neurosci 2009; 12: 1248-1256.

26 Bosenberg M, Muthusamy V, Curley DP, Wang Z, Hobbs C, Nelson B et al. Characterization of melanocyte-specific inducible Cre recombinase transgenic mice. Genesis 2006; 44: 262-267.

27 Damsky WE, Curley DP, Santhanakrishnan M, Rosenbaum LE, Platt JT, Gould Rothberg BE et al. Beta-catenin signaling controls metastasis in Braf-activated Pten-deficient melanomas. Cancer Cell 2011; 20: 741-754.

28 Akasaka K, Maesawa C, Shibazaki M, Maeda F, Takahashi K, Akasaka T et al. Loss of class III beta-tubulin induced by histone deacetylation is associated with chemosensitivity to paclitaxel in malignant melanoma cells. J Invest Dermatol 2009; 129: 1516-1526.

29 Talantov D, Mazumder A, Yu JX, Briggs T, Jiang Y, Backus J et al. Novel genes associated with malignant melanoma but not benign melanocytic lesions. Clin Cancer Res 2005; 11: 7234-7242.

30 Riker Al, Enkemann SA, Fodstad O, Liu S, Ren S, Morris C et al. The gene expression profiles of primary and metastatic melanoma yields a transition point of tumor progression and metastasis. BMC Med Genomics 2008; 1: 13.

31 Singh S, Trevino J, Bora-Singhal N, Coppola D, Haura E, Altiok S et al. EGFR/Src/Akt signaling modulates Sox2 expression and self-renewal of stem-like side-population cells in non-small cell lung cancer. Mol Cancer 2012; 11: 73.

32 Lundberg IV, Burstrom AL, Edin S, Eklof V, Oberg A, Stenling R et al. SOX2 expression is regulated by BRAF and contributes to poor patient prognosis in colorectal cancer. PloS One 2014; 9: e101957.

33 Graziani G, Artuso S, De Luca A, Muzi A, Rotili D, Scimeca M et al. A new water soluble MAPK activator exerts antitumor activity in melanoma cells resistant to the BRAF inhibitor vemurafenib. Biochem Pharmacol 2015; 95: 16-27.

34 Lacal PM, Ruffini F, Pagani E, D'Atri S. An autocrine loop directed by the vascular endothelial growth factor promotes invasiveness of human melanoma cells. Int $J$ Oncol 2005; 27: 1625-1632.

35 Sanchez-Laorden B, Viros A, Girotti MR, Pedersen M, Saturno G, Zambon A et al. BRAF inhibitors induce metastasis in RAS mutant or inhibitorresistant melanoma cells by reactivating MEK and ERK signaling. Sci Signal 2014; 7: ra30.

36 Leis O, Eguiara A, Lopez-Arribillaga E, Alberdi MJ, Hernandez-Garcia S, Elorriaga K et al. Sox 2 expression in breast tumours and activation in breast cancer stem cells. Oncogene 2012; 31: 1354-1365.

37 Hutz K, Mejias-Luque R, Farsakova K, Ogris M, Krebs S, Anton M et al. The stem cell factor SOX2 regulates the tumorigenic potential in human gastric cancer cells. Carcinogenesis 2014; 35: 942-950.

38 Camilo V, Barros R, Celestino R, Castro P, Vieira J, Teixeira MR et al. Immunohistochemical molecular phenotypes of gastric cancer based on SOX2 and CDX2 predict patient outcome. BMC Cancer 2014; 14: 753.

39 Simon FS, Segalad C, Cheng P, Bonalli M, Parfejevs V, Levesque M et al. Sox2 is dispensable for primary melanoma and metastasis formation. Oncogene; e-pub ahead of print 3 April 2017.

40 Graham V, Khudyakov J, Ellis P, Pevny L. SOX2 functions to maintain neural progenitor identity. Neuron 2003; 39: 749-765.

41 Wood HB, Episkopou V. Comparative expression of the mouse Sox1, Sox2 and Sox3 genes from pre-gastrulation to early somite stages. Mech Dev 1999; 86: 197-201.

42 Shakhova O, Zingg D, Schaefer SM, Hari L, Civenni G, Blunschi J et al. Sox10 promotes the formation and maintenance of giant congenital naevi and melanoma. Nat Cell Biol 2012; 14: 882-890.

43 Dankort D, Filenova E, Collado M, Serrano M, Jones K, McMahon M. A new mouse model to explore the initiation, progression, and therapy of BRAFV600E-induced lung tumors. Genes Dev 2007; 21: 379-384. 